\title{
OPTIMAL STRATEGY FOR THE VARDI CASINO WITH INTEREST PAYMENTS
}

\author{
ILIE GRIGORESCU*** AND \\ ROBERT CHEN, ${ }^{* * * *}$ University of Miami \\ LARRY SHEPP, ${ }^{* * * *}$ Rutgers University
}

\begin{abstract}
A gambler starts with fortune $f<1$ and plays in a Vardi casino with infinitely many tables indexed by their odds, $r \geq 0$. In addition, all tables return the same expected winnings per dollar, $c<0$, and a discount factor is applied after each round. We determine the optimal probability of reaching fortune 1 , as well as an optimal strategy that is different from bold play for fortunes larger than a critical value depending exclusively on $c$ and $1+a$, the discount factor. The general result is computed explicitly for some relevant special cases. The question of whether bold play is an optimal strategy is discussed for various choices of the parameters.
\end{abstract}

Keywords: Gambling problem; Vardi casino; optimal strategy; bold play

2000 Mathematics Subject Classification: Primary 60G40

Secondary 91A60

\section{Introduction}

The main result of the paper is Theorem 1, which states the optimal probability, $P_{0}(f)$, of reaching wealth of at least 1 (nonextinction) when we start with wealth $f, 0 \leq f \leq 1$, in a casino with a continuum range of odds $r \in \mathcal{R}$, at tables indexed by $r \geq 0$, with expected winnings per dollar equal to $c \in(-1,0)$ at every table and inflation (or interest) rate $a>0$. More precisely, a gambler starts off with wealth $f$ and at any table of the casino is allowed to stake an amount $s$ with the restrictions that $0 \leq s \leq f$ and $f+r s \leq 1+a$. The latter condition can be ignored because, as we will show, it is never violated for any optimizing strategy. Tables are indexed by their odds, $r$, meaning that a stake $s$ at table $r$ is lost with probability $1-w$ and returns $r s$ if, with probability $w$, the gambler wins. In this paper, every time a game is played, the current wealth is discounted by a factor $(1+a)^{-1}$, accounting for inflation (or interest). It is probably true that the optimal strategy is unique for $a>0$, but for $a=0$ this is false.

What we mean by a Dubins $(r, c)$ casino is a casino with only one table, this giving odds $r$ and returning expected payoff $c<0$ on a dollar bet (also known as a subfair casino). Dubins and Savage [6] considered the more general case where the casino has several tables, but they do not seem to have considered the casino proposed by Vardi [13], where a table, $T_{r}$, is available for every odds $r$ and $c$ is fixed and has the same negative value at all the tables. Such a casino will

Received 23 May 2006; revision received 6 October 2006.

* Postal address: Department of Mathematics, University of Miami, Coral Gables, FL 33124-4250, USA.

** Email address: igrigore@math.miami.edu

*** Email address: chen@math.miami.edu

**** Postal address: Department of Statistics, Rutgers University, Piscataway, NJ 08855, USA.

Email address: shepp@stat.rutgers.edu 
hereafter be called a Vardi casino, with or without interest, respectively according to whether $a>0$ or $a=0$. This terminology was introduced in [12].

The expected payoff, $c$, is equal to $(+1) r w+(-1)(1-w)<0$, which implies that (throughout the paper) $w \equiv w(c, r)=(1+c) /(1+r)$. It thus provides an upper bound on the optimal probability of reaching fortune 1 in any casino if $c$ is the largest expected return on any of the tables. It is shown by John Lou in a forthcoming thesis [9] that having all the additional tables and odds provided in the Vardi casino yields only a relatively small gain in the optimal probability of reaching fortune 1 over that of the Dubins casino, which seems quite surprising.

All tables are independent of each other, and all games at each table are independent of each other. More formally, let $\Omega=\{-1,+1\}^{\mathcal{R} \times \mathbb{Z}_{+}}$with the $\sigma$-field $\mathcal{F}$ generated by cylinder functions and $\mathcal{F}_{n}$ denoting the sequences of outcomes for all tables up to time $n$. Since $\Omega=\bigotimes_{r \in \mathcal{R}} \Omega_{r}$, where $\Omega_{r}=\{\omega(r, \cdot): \omega \in \Omega\}$ are the projections of $\Omega$, we introduce Bernoulli measures $\mathrm{P}_{r}$ on $\Omega_{r}$ assigning probability $w(c, r)=(1+c) /(1+r)$ to +1 and probability $1-w(c, r)$ to -1 for all $n$, and write $\mathbb{P}=\bigotimes_{r \in \mathcal{R}} \mathrm{P}_{r}$ for their infinite product.

A gambling strategy, or simply a strategy, $S$ is a sequence of measurable functions

$$
S_{n}(\omega, \cdot):[0,1] \rightarrow[0,1] \times \mathcal{R}, \quad S_{n}(\omega, f)=(s, r), \quad n \geq 0, \omega \in \Omega,
$$

adapted to the filtration $\left\{\mathcal{F}_{n}\right\}_{n \geq 0}$, that assign a pair $(s, r) \equiv\left(s_{n}(\omega, f), r_{n}(\omega, f)\right)=S_{n}(\omega, f)$ to every value $f \in[0,1]$ current at time $n$, with the single restriction that

$$
0 \leq s_{n}(\omega, f) \leq f
$$

The set of strategies will be denoted by $\&$.

In other words, strategy $S$ tells the gambler how much he should bet and at which table, for a given fortune, at a given time. More general strategies than Markovian strategies do not provide any additional probability of reaching fortune 1 . In fact, we show that all optimal strategies discussed here are simply functions of $f$, not of $\omega$ and $n$. In other words, all the optimal strategies discussed here are Markovian. In the following, we shall omit ' $\omega$ ' from $S_{n}(\omega, f)$, and we shall use the convention that we omit the subscript ' $n$ ' whenever $S_{n}(f)$ depends only on $f$.

We assume that $\mathcal{R}=[0, \infty)$, but it is interesting to put the present results in the context of various other choices of $\mathcal{R}$. The classical result of Dubins and Savage [6] (see also [10] for more background on the problem) showed that, when $a=0$ and $\mathcal{R}=\{r\}$, the optimal strategy is bold play: more precisely, $S_{b}(f)=f$ when $f \leq(1+r)^{-1}$ and $S_{b}(f)=(1-f) / r$ when $f>(1+r)^{-1}$ (use the maximum bet allowed at any time). The bold-play conjecture dating back to Coolidge [5] (see also [4] for more comments on this) is not valid for a Vardi casino without inflation, as shown in [12], where the parameters are $a=0$ and $\mathcal{R}=[0, \infty)$. Nonoptimality of bold play in a one-table casino in the presence of inflation was proved in earlier work [1], [2], [8] and in a different setting in [7]. For a subfair primitive casino with one table, this satisfying the condition that $1 / r \leq a \leq r$, for $r>1$ Chen et al. [3] showed that bold play is not optimal, yet conjectured for $r<1$ that it is. A recent result [4] proves the conjecture under the additional assumption that $w \leq \frac{1}{2}$.

The present article sheds some light on the interplay between various parameters defining the casino, and on when bold play is optimal. We give a complete answer to the problem in the case in which $a>0$ and $\mathcal{R}=[0, \infty)$. As anticipated from the preceding discussion, a dichotomy between the bold- and nonbold-play regimes emerges, depending on the choice of parameters 
$(c, a)$. There exists a value, $\hat{f}$, such that the optimal strategy is strict bold play $(s(f)=f)$ for $f \leq \hat{f}$ and a more cautious policy is required for $f>\hat{f}$ (see (17) for a particular case). Some parameter combinations, such as those for which $a^{2}>|c|$ (see Section 2), have $\hat{f}=1$, allowing bold play only, in some sense concealing the nature of the general problem. Another remarkable feature of the solution is the presence of a jump at $f=1$, meaning that if $a>0$ then the extinction probability is bounded away from 0 even as $f$ tends to 1 , showing that the gambler cannot beat inflation, even under optimal play. An interesting question is: what effect would a random inflation rate have on the discontinuity at $f=1$ ?

The authors believe that the present constructive approach based on the variational formulae (12) and (14) from Theorem 1 can settle the other cases, when various subsets of $[0, \infty)$ are used for $\mathcal{R}$ in the definition of the casino.

Let $\phi$ denote a continuous function on [0,1]. For a given strategy $S$, let $\left\{X_{n}^{S}\right\}_{n \geq 0}$ be the discrete-time stochastic process representing the fortunes at times $n=0,1, \ldots$ under strategy $S$. The chain starts at $X_{0}^{S}=f \in[0,1]$, is adapted to $\left\{\mathcal{F}_{n}\right\}_{n \geq 0}$, and satisfies the relation

$$
\mathbb{E}\left(\phi\left(X_{n+1}^{S}\right) \mid X_{n}^{S}=g\right)= \begin{cases}w\left(c, r_{n}(g)\right) \phi\left(\frac{g+r_{n} s_{n}(g)}{1+a}\right) & \\ +\left(1-w\left(c, r_{n}(g)\right)\right) \phi\left(\frac{g-s_{n}(g)}{1+a}\right), & g \in(0,1), \\ \phi(g), & g=0,1 .\end{cases}
$$

We note that $X_{n}$ is not Markovian in general, as the strategy $S$ may take into account the whole past.

The chain $\left\{X_{n}^{S}\right\}_{n \geq 0}$ is bounded above and below and is a supermartingale with respect to $\left\{\mathcal{F}_{n}\right\}_{n \geq 0}$ (see Theorem 1), as a consequence of the subfair nature of the casino. With probability 1, the $\operatorname{limit}_{n \rightarrow \infty} X_{n}^{S}$ exists, and we denote it by $X_{\infty}^{S}$. Let $P_{f}^{S}$ be the probability of reaching fortune 1 before extinction, starting with initial fortune $f$, while applying the strategy $S$, with (recall) $P_{0}(f)$ being the optimal probability of doing so, i.e.

$$
P_{f}^{S}=\mathbb{P}\left(X_{\infty}^{S}=1 \mid X_{0}^{S}=f\right), \quad P_{0}(f)=\sup _{S \in S} P_{f}^{S} .
$$

Our goal is to determine $P_{0}(f)$ for any $f \in[0,1]$ and to formulate at least one strategy to achieve it.

Strict bold play is the strategy consisting of staking all the gambler's fortune for any $f \in$ $(0,1)$. Of course, this strategy is never optimal if $a$ is small, since it is foolish to exceed fortune 1 .

It is intuitively clear that, for large $a$, (i) the gambler will be forced to bet all his wealth $f$, for all $f \in(0,1)$ (strict bold play), and (ii) the optimal strategy has a gap at $f=1$, i.e. $P_{0}(1-)<1$. This behavior contrasts with the zero-interest rate setting $(a=0)$, where, as was proved in [12], $P_{0}(f)=1-(1-f)^{1+c}$. In Section 2 we present the special case in which $a$ drops below the critical value, $\sqrt{|c|}$, in which case strategy (i) applies. While this is covered by the general result from Theorem 1, it is shown directly in Proposition 1.

In Section 3 we prove the general result. The optimal probability is defined via a variational formula, (12), for $R(f)=1-P_{0}(f)$, and is shown to be convex in (14). The discontinuity at $f=1$ is consistent with the fact that $P_{0}(f)$ is lower semicontinuous.

For general parameters $(a, c)$, the case in which $a^{2}<|c|$ is more complex than that in which $a^{2} \geq|c|$, since $P_{0}(f)$ is not given explicitly, even though it is technically speaking fully 
computable, not just numerically. This is because the infimum in (12) is a finite-dimensional problem, since the number (see (10)) of parameters, $k \leq n(a, c)<\infty$, is a constant dependent on $a$ and $c$ but not on $f$. For a relevant particular choice of parameters $(a, c)$ such that $k=1$, in Section 4 we derive explicit expressions (see (17)) for $P_{0}(f)$ and the discontinuity at $f=1$.

Finally, in Section 6 we provide an upper bound (see $(22)$ ) for $P_{0}(f)$, equal to a smooth perturbation of the result of [12], which corresponds to $a=0$. This approximation does not exhibit the discontinuity at $f=1$, making it useful in the intermediate parameter range above the bold-play range yet away from 1 for pairs $(a, c)$ with large $n(a, c)$.

The gambler with current fortune $f$ is allowed to bet $s$ dollars on a table with $r \geq(1+$ $a-f) / s$. If he wins, his new fortune will exceed $f=1$ before application of the discount $(1+a)^{-1}$. However, if his strategy is optimal then the strategy with $r=(1+a-f) / s$ would be optimal as well, since the value of $r$ does not matter when the gambler loses, according to $(2)$.

In addition, given that the gambler will stop when his fortune reaches either 0 or 1 , the strategies can be defined arbitrarily at $f=0$ and $f=1$. We adopt the natural choice $s_{n}(0)=s_{n}(1)=0, n \geq 0$, and can choose $r$ arbitrarily, since for fortune $f=0$ or $f=1$ the gambler does not actually play the next game. Notice that in the absence of inflation $(a=0)$, it would be enough to specify that $s=0$, whereas for $a>0$ even passively waiting a turn and not playing reduces the fortune to $f /(1+a)$. Without loss of generality, we shall assume throughout the paper that any strategy $S$ satisfies

$$
0 \leq s_{n}(f) \leq f, \quad r_{n}(f) s_{n}(f) \leq 1+a-f, \quad s_{n}(0)=s_{n}(1)=0,
$$

for any $n \geq 0$. For simplicity, we shall use the notation $p=(1+c) /(1+a)$.

\section{The case $a^{2} \geq|c|$}

We prove that the optimal probability of survival (see (3)) is achieved by the strict bold-play strategy $S_{\mathrm{sb}}(f)=(f,(1+a-f) / f), f \in(0,1)$, and is equal to

$$
P_{0}(f)= \begin{cases}p f, & f<1 \\ 1, & f=1 .\end{cases}
$$

Proposition 1. For all $f$ with $0 \leq f \leq 1$, s with $0 \leq s \leq f$, and $r \geq 0$ with $r s \leq 1+a-f$, the function $P_{0}(f)$ satisfies

$$
P_{0}\left(\frac{f+r s}{1+a}\right) w(c, r)+P_{0}\left(\frac{f-s}{1+a}\right)(1-w(c, r)) \leq P_{0}(f) .
$$

Proof. We have to prove (6) for $f<1$. For $f=1$ the inequality is trivial, since $s=0$.

If $(f+r s) /(1+a)<1$ then the inequality is equivalent to $s c \leq a f$, which is evidently true since $c<0$. If $(f+r s) /(1+a)=1$, then the inequality becomes

$$
w+p\left(\frac{f-s}{1+a}\right)(1-w) \leq p f, \quad \text { with } \quad w=\frac{1+c}{1+r} .
$$

Fix $r \geq 0$ and regard (7) as an inequality in $f$. The restriction that $1+a=f+r s$ makes sense only if $r \geq a$. In addition, we must have $f \geq(1+a) /(1+r)$. Moving all the terms to the 
right-hand side of the inequality yields an expression linear in $f$. It is sufficient to verify the inequality at the endpoints. For $f=(1+a) /(1+r)$, it is easy to verify. For $f=1$, it reads

$$
\frac{1+c}{1+r}+\frac{1+c}{1+a}\left(\frac{1+r}{r(1+a)}-\frac{1}{r}\right)\left(1-\frac{1+c}{1+r}\right) \leq \frac{1+c}{1+a},
$$

which is equivalent to $(r-a)(r a-|c|) \geq 0$. The restrictions on the parameters mean that $r \geq a$, concluding the proof.

Remark 1. The range of $r$ is indeed arbitrarily close to $a$ for bold play; this can be seen by taking $f=s=1-\varepsilon$ and $r=(1+a-f) / f$ for arbitrary $\varepsilon>0$.

Proposition 2. Strict bold play, that is, betting the full wealth $f, 0<f<1$, on the table with odds $r=(1+a-f) / f$, achieves the probability of survival $P_{0}(f)$ in (5).

Proof. We see by conditioning on the outcome of the first game that, for all $f \in(0,1)$, the probability, $P(f)$, of reaching wealth 1 when starting with wealth $f$ under strict bold play satisfies

$$
P(f)=\frac{1+c}{1+r} P\left(\frac{1+r}{1+a} f\right)=\frac{1+c}{1+(1+a-f) / f} P(1)=P_{0}(f) .
$$

\section{The general case}

In the following we shall use the notation $\eta=\left(a+a^{2}\right) /(a+|c|)$.

Proposition 3. Let $\hat{f}$ be the largest value of $f \leq 1$ for which (6) is satisfied for any choice of $s$ and $r$ admissible in the sense of (4). Then $\hat{f}=1$ for $a^{2} \geq|c|$ and $\hat{f}=\eta$ for $a^{2}<|c|$.

Proof. Proposition 1 proved that if $a^{2} \geq|c|$ then (6) is satisfied for all $f \leq 1$, showing that $\hat{f}=1$ in this case.

If $a^{2}<|c|$ then, following the steps of the proof of Proposition 1, we see that (6) is satisfied automatically for $(f+r s) /(1+a)<1$. For $f+r s=1+a$, (6) becomes (7). The restrictions in (4) imply that $f \geq(1+r) /(1+a)$. The easiest way to check this is to plot $(s, t)$ with all other parameters fixed and see that the domain defined by $0 \leq s \leq f$ and $f+r s \leq 1+a$ has vertices $(0,0),(0,1+a)$, and $((1+r) /(1+a),(1+r) /(1+a))$. We rewrite $(7)$ as

$$
\frac{r a-|c|}{r(1+a)}\left(f-\frac{1+a}{1+r}\right) \geq 0 .
$$

Let $r^{\prime}=|c| / a$. If $f \leq \eta$ then $1+r^{\prime}=(1+a) / \eta \leq(1+a) / f \leq 1+r$, so (9) is satisfied, showing that $\hat{f} \geq \eta$. However, for $f>\eta$ there exist admissible values of $r$ such that (9) is not satisfied, implying that $\hat{f} \leq \eta$.

Remark 2. The table with index $r^{\prime}=|c| / a$ appearing in (8) and (9) corresponds to the critical value $\hat{f}=\eta$, and can be achieved under bold play with the bet $s^{\prime}=\eta$.

Propositions 1,2, and 3 suggest that there must be two regimes of play, respectively according to whether the current fortune $f$ is above or below the critical value $\hat{f}=\eta$. Assuming that we start with $f>\eta$, we shall look at sequences of descending fortunes $f_{j}$ obtained for consecutive unsuccessful bets. In general, the only restriction is that $(1+a) f_{j}=f_{j-1}-s_{j-1} \leq f_{j-1}$. Once the fortune drops below $\eta$, we intuitively know that the optimal strategy is bold play. Finally, the optimal strategy is obtained by optimizing over all scenarios (descending sequences) leading to a fortune below $\eta$. We now formalize these ideas, starting with a definition. 
Definition 1. Let $f \in[0,1)$. A descending sequence for $f$ of length $k+1$ is a sequence of fortunes $f_{j}, j=0,1, \ldots, k$, such that (i) $f_{0}=f$, (ii) $k=0$ if $f \leq \eta$, (iii) $f_{j} \leq(1+a)^{-1} f_{j-1}$, $j=1, \ldots, k$, and (iv) $f_{k-1}>\eta$ and $f_{k} \leq \eta$. Such a sequence will be denoted by $\{f\}$, the set of descending sequences will be denoted by $D(f)$, and the set of descending sequences of length $k$ will be denoted by $D_{k}(f)$.

A descending sequence has finite length for any $f$. The maximum admissible length, $k_{\max }$, is bounded above by

$$
n(a, c)=-\frac{\ln \eta}{\ln (1+a)}+1=\frac{\ln (a+|c|)-\ln \left(a+a^{2}\right)}{\ln (1+a)}+1,
$$

a constant depending exclusively on $a$ and $c$. Recall that $p=(1+c) /(1+a)$.

For every $f$ and every $\{f\} \in D_{k}(f)$, we construct the function

$$
R^{\{f\}}(f)=\left(1-p f_{k}\right) \prod_{j=0}^{k-1}\left(1-p \frac{f_{j}-(1+a) f_{j+1}}{1-f_{j+1}}\right),
$$

with the convention that $R^{\{f\}}(f)=(1-p f)$ for $f \leq \eta$, which is consistent with Definition 1 .

We notice that $0 \leq R^{\{f\}}(f) \leq 1$ and define the function $P_{0}(f)$ by $P_{0}(f)=1$ for $f=1$ and by

$$
P_{0}(f)=1-R(f), \quad \text { with } \quad R(f)=\inf _{k} \inf _{\{f\} \in D_{k}(f)} R^{\{f\}}(f),
$$

for $f<1$.

The infimum is achieved at least for a certain $k \equiv k(f)$ and a certain $\{f\} \in D_{k}(f)$. To see this, we recall that $k \leq n(a, c)$ has a finite range independent of $f$. Equation (12) shows that the functions $R^{\{f\}}(f)$ can be written as continuous functions of the $k+1$ variables $f_{0}, f_{1}, \ldots, f_{k}$ on a compact domain associated with $f$. For each $f$, we choose one of the minimizing sequences of (12) and denote it by $\{f\}^{-}$. Then evidently $R(f)=R^{\{f\}^{-}}(f)$.

Proposition 4. The function $P_{0}(f)$ is convex in $f$.

Proof. We have to show that $R(f)$ is concave. As defined in (12), the infimum is taken over a set depending on $f$ itself; we shall write it in a form that clearly shows that $R(f)$ is the infimum of a family of linear functions over a set independent of $f$. For a pair $\left(f_{j}, f_{j+1}\right)$, let $r_{j}$ and $\gamma_{j}$ be defined by the equality

$$
\gamma_{j}=\frac{f_{j}-(1+a) f_{j+1}}{1-f_{j+1}}=\frac{1+a}{1+r_{j}} .
$$

We can interpret $f_{j+1}=\left(f_{j}-s_{j}\right) /(1+a)$ as the result of losing a bet $s_{j}$ at the table $r_{j}$, chosen such that a winning bet would have increased the fortune to exactly 1 , i.e. $\left(f_{j}+r_{j} s_{j}\right) /(1+a)=1$ (bold play). Then

$$
r_{j}=\frac{1+a-f_{j}}{s_{j}} \geq \frac{1+a-f_{j}}{f_{j}} \geq a .
$$

With this in mind, the sequence of $\gamma_{j}$ defines a finite number of parameters in $(0,1]$, without other restrictions depending on $f$. 
To make the computation easier, let $\alpha_{j}=\left(1+a-\gamma_{j}\right)^{-1}$ and $\beta_{j}=\gamma_{j} \alpha_{j}$. Then

$$
\begin{aligned}
f_{1} & =\alpha_{0} f_{0}-\beta_{0}, \\
& \vdots \\
f_{k-1} & =\alpha_{k-2} f_{k-2}-\beta_{k-2}, \\
f_{k} & =\alpha_{k-1} f_{k-1}-\beta_{k-1}
\end{aligned}
$$

and

$$
f_{k}=A f-B \quad \text { with } \quad A=\prod_{j=0}^{k-1} \alpha_{j}, \quad B=\sum_{j=0}^{k-1} \beta_{j} \prod_{i=j+1}^{k-1} \alpha_{i} .
$$

Recasting (see (12)) in terms of the independent $\left\{\gamma_{j}\right\}_{0 \leq j \leq k-1}, 0<\gamma_{j} \leq 1$, and $f$, we have

$$
R(f)=\inf _{k} \inf _{\{\gamma\} \in(0,1]^{k}}(1+p B-p A f) \prod_{j=0}^{k-1}\left(1-p \gamma_{j}\right),
$$

where the first factor is a linear function of $f$, as in (13). This proves that $R(f)$ is concave (see [11]).

In the notation of Definition 1, for every $\{f\} \in D(f)$ we define the sequence of bets $\{s\}=\left\{s_{0}, s_{1}, \ldots, s_{k}\right\}$ as follows, according to the sequence length $k(f)$ : if $k(f)=1$ then $s_{0}=f_{0}$, and if $k(f)>1$ then $s_{j}=f_{j}-(1+a) f_{j+1}, j=0, \ldots, k-1$, with $s_{k}=f_{k}$. We denote by $\{s\}^{-}$the sequence corresponding to $\{f\}^{-}$.

Definition 2. Let $S^{-}$be a strategy defined as follows. For $f=0$ or $f=1$, we stop. For $0<f<1$, we have two alternatives.

1. If $f \leq \eta$ then we bet $s(f)=f$ on the table with $r(f)=(1+a-f) / s(f)$; if we win then we have reached 1 and we stop, and if we lose then we stop as well, since $f-s(f)=0$.

2. If $f>\eta$ then we generate a minimizing descending sequence $\{f\}^{-}=\left\{f_{0}^{-}, f_{1}^{-}, \ldots, f_{k}^{-}\right\}$, $k \equiv k(f)$, starting with $f_{0}^{-}=f$ and ending with $f_{k}^{-} \leq \eta$ (by construction), with the corresponding sequence of bets $\{s\}^{-}=\left\{s_{0}^{-}, s_{1}^{-}, \ldots, s_{k}^{-}\right\}$. We first bet $s(f)=f_{0}^{-}$ $(1+a) f_{1}^{-}$on the table with $r \equiv r(f)=(1+a-f) / s(f)$; if we win then we stop, and if we lose then we proceed by betting $s\left(f_{1}^{-}\right)=s_{1}^{-}$, and so on, until we either win (and stop) or reach $f_{k(f)}^{-} \leq \eta$. If we reach $f_{k(f)}^{-} \leq \eta$ then we set $f=f_{k(f)}^{-}$and go to alternative 1 .

Theorem 1. The optimal probability of reaching fortune 1 when we start with wealth $f, 0 \leq$ $f \leq 1$, is $P_{0}(f)=1-R(f)$ if $f<1$ and $P_{0}(f)=1$ if $f=1$, and is realized by the strategy $S^{-}$. In addition, $1-P_{0}(1-) \geq(1-p)^{n(a, c)+1}>0$, where $n(a, c)$ is the bound in (10).

The theorem will be proved in three steps: in Proposition 5 we show that the strategy $S^{-}$ yields $P_{0}(f)=1-R(f)$ for $f<1$, in Proposition 6 we show that $P_{0}(f)$ is an upper bound on the probability of reaching fortune 1 , and finally we prove the lower bound for the discontinuity at $f=1$.

Proposition 5. The probability of survival $P_{f}^{S^{-}}$defined in (3), starting from $f<1$ and corresponding to the strategy $S^{-}$from Definition 2 , is equal to $P_{0}(f)$. 
Proof. Let $R^{-}(f)=1-P_{f}^{S^{-}}$, let $f=f_{0}$, and let $f_{1}^{-}$be the wealth in case of loss while applying $S^{-}$(in one step). By conditioning upon the events of winning and losing in the first play, the law of total probability and (2) applied to the chain $X_{n}^{S^{-}}$yield for $R^{-}(f)$ the recurrence relation

$$
R^{-}\left(f_{0}\right)=\left(1-p \frac{f_{0}-(1+a) f_{1}^{-}}{1-f_{1}^{-}}\right) R^{-}\left(f_{1}^{-}\right) .
$$

To see this, we remember that

$$
\frac{f_{0}-(1+a) f_{1}^{-}}{1-f_{1}^{-}}=\frac{1+a}{1+r_{1}^{-}}, \quad r_{1}^{-}=r\left(f_{0}\right),
$$

according to Definition 2. Note that, for $f \leq \eta,(15)$ leads trivially to equality between $R^{-}(f)$ and $R(f)$, since $f_{1}^{-}=0$. For $f>\eta$, (15) is satisfied by $R(f)$ once again, as seen in (11) applied to the optimizing sequence. We can repeat this reasoning for $f_{2}^{-}, f_{3}^{-}$, and so on, to see that $R^{-}(f)=R(f)$. Alternatively, if $\{f\}^{-}$is an optimal descending sequence for $f$ and $f_{1}^{-}$is the second term in the sequence, then the truncated sequence $\left\{f_{1}^{-}\right\}=\left\{f_{1}^{-}, f_{2}^{-}, \ldots, f_{k}^{-}\right\}$, that is, the same sequence shifted by one unit, is an optimal descending sequence for $f_{1}^{-}$. This fact is clear by construction. We have thus shown that $R^{-}(f)=R(f)$.

Proposition 6. For any given $(a, c)$ and any triple $(f, s, r)$ compatible in the sense of (4), with $0 \leq f<1,0 \leq s \leq f$, and $r \geq 0$, the function $P_{0}$ in (12) satisfies the inequality

$$
P_{0}\left(\frac{f+r s}{1+a}\right) w+P_{0}\left(\frac{f-s}{1+a}\right)(1-w) \leq P_{0}(f) .
$$

We note that $f=1$ implies that $s=0$, in which case (16) is trivial.

Proof of Proposition 6. By construction, the function $P_{0}(f)$ is convex, being the supremum over linear functions of $f$, according to Proposition 4. As functions of $s$, both $P_{0}((f+$ $r s) /(1+a))$ and $P_{0}((f-s) /(1+a))$ are convex, so the left-hand side of $(16)$ is convex in $s$. The maximum can only be achieved at extreme values of $s$. Given the restrictions on $s$,

(i) if $f>(1+a) /(1+r)$ then the extreme values are $s=0$ and $s=(1+a-f) / r$, and

(ii) if $f \leq(1+a) /(1+r)$ then the extreme values are $s=0$ and $s=f$.

Suppose that $s=0$ in either case (i) or case (ii). If $f \leq \eta$ then we recover (6), and the proof is the same. If $f>\eta$ then $f_{0}=f$ and $f_{1}=f /(1+a)$ can be seen to be the first two admissible terms of a descending sequence $\{f\}$. By construction, $P_{0}\left(f_{1}\right) \leq P_{0}\left(f_{0}\right)$.

Now suppose that $s=(1+a-f) / r$ in case (i). This corresponds to bold play, that is, $r$ is such that we reach fortune 1 if we win. We have to prove that $R(f) \leq R((f-s) /(1+a))(1-w)$. Writing $f_{0}=f$ and $f_{1}=(f-s) /(1+a)$, we have the equivalent inequality

$$
R(f) \leq\left(1-p \frac{f_{0}-(1+a) f_{1}}{1-f_{1}}\right) R\left(f_{1}\right),
$$

which is evidently true by construction.

Finally, suppose that $s=f$ in case (ii). We can reduce the problem to showing that $R(f) \leq R(f(1+r) /(1+a)) w+(1-w)$ or, equivalently, that

$$
P_{0}\left(\frac{1+r}{1+a} f\right) w \leq P_{0}(f)
$$


for all $f$ and $r$ satisfying $0 \leq r \leq(1+a-f) / f=(1+a) / f-1$ (since $s=f)$. Rewrite the desired inequality as

$$
(1+c) P_{0}\left(\frac{1+r}{1+a} f\right)-(1+r) P_{0}(f) \leq 0 .
$$

Let $f$ be fixed. As a function of $r$, the left-hand side is convex. The maximum is achieved at one of the endpoints. At $r=0$ we have

$$
P_{0}(f) \geq P_{0}\left(\frac{1}{1+a} f\right)(1-|c|),
$$

which is weaker than $P_{0}(f) \geq P_{0}(f /(1+a))$ or, equivalently, $R(f) \leq R(f /(1+a))$. This inequality is true for any $f<1$ by construction, taking $f_{0}=f$ and $f_{1}=f /(1+a)$ as the first two terms of a descending sequence for $f$ if $f>\eta$, and simply verifying it directly if $f \leq \eta$. Finally, at the upper endpoint, $r=(1+a) / f-1$, for which $f(1+r) /(1+a)=1$, the inequality becomes $R(f) \leq 1-p f$. This is true by construction, by taking $f_{0}=f$ and $f_{1}=0$ in a descending sequence for $f$.

Proof of Theorem 1. Denote by $\left\{X_{n}^{S}\right\}_{n \geq 0}$ the sequence of the values at times $n=0,1, \ldots$ of the gambler's wealth under an admissible strategy $S \in \&$, satisfying (2). We drop the superscript ' $S$ ' since there is no possibility of confusion. At time $n$, the gambler chooses a stake and a table corresponding to the current value of his fortune, $X_{n}$, according to $\left\{\left(s_{n}\left(X_{n}\right), r_{n}\left(X_{n}\right)\right)\right\}_{n \geq 0}$ with the convention that $X_{n}$ stays at 0 or 1 once it has reached either value for the first time. Moreover, since $\left\{X_{n}\right\}$ is bounded by 1 , it is easy to check that it is a supermartingale. Inequality (16) and the fact that $P_{0}$ is bounded show that $P_{0}\left(X_{n}\right)$ is also a supermartingale. The limit, $X_{\infty}$, of $X_{n}$ as $n \rightarrow \infty$ exists almost surely. Since $P_{0}(0)=0, P_{0}(1)=1$, and $\mathbf{1}_{[1, \infty)}(x) \leq P_{0}(x)$, we have

$$
\mathbb{P}\left(X_{\infty}=1 \mid X_{0}=f\right) \leq \mathbb{E}\left(P_{0}\left(X_{\infty}\right) \mid X_{0}=f\right) \leq \mathbb{E}\left(P_{0}\left(X_{0}\right) \mid X_{0}=f\right)=P_{0}(f) .
$$

However, Proposition 5 shows that $\mathbb{P}\left(X_{\infty}=1 \mid X_{0}=f\right) \geq P_{0}(f)$, by applying strategy $S^{-}$.

Finally, it remains to show that $P_{0}(1-)<1$ or, equivalently, that $R(1-)>0$. Note that the product (11) has at most $k+1$ factors, each bounded below by $1-p$, and that $k$ is bounded above by a value depending on $a$ and $c$ only. This concludes the proof.

\section{Explicit results for $k(f) \leq 1$}

For $a^{2} \leq|c| \leq 2 a^{2}+a^{3}$, we shall see that the descending sequence from Definition 1 has length $k \leq 1$ and an explicit form of the optimum function $P_{0}(f)$ can be derived. In fact, this inequality between $a$ and $c$ is equivalent to having $k(f) \leq 1$ for all $f$. Let $f_{0}=f$ and $f_{1}<f_{0}$ be such that $f_{1} \leq(1+a)^{-1} f_{0}$. It is easy to see that $f_{1} \leq \eta$ if $2 a^{2}+a^{3} \geq|c|$, for any initial $f$. On the other hand, let us assume that the second term satisfies $f_{1} \leq \eta$ for any $f$. We want to prove that $2 a^{2}+a^{3} \geq|c|$. Since $s=f_{0}-(1+a) f_{1}$, we introduce $r$, the table where we bet under the restriction that $f+r s=1+a$ (bold play), and obtain,

$$
f_{1}=\frac{1+r}{r(1+a)} f_{0}-\frac{1}{r}
$$

The condition $k(f) \leq 1$ is equivalent to

$$
\frac{1+r}{r(1+a)} f-\frac{1}{r} \leq \frac{a+a^{2}}{a+|c|},
$$


which reduces to

$$
\frac{1}{r} \geq \frac{(1+a)\left(|c|-2 a^{2}-a^{3}\right)}{a(a+|c|)(1+a)}
$$

satisfied by any $r$ given that

$$
a^{2} \leq|c| \leq 2 a^{2}+a^{3} .
$$

The left-hand side of this inequality is not required to define the strategy, but was included to underscore the interval in which $r$ is located.

Proposition 7. If $a^{2}<|c| \leq 2 a^{2}+a^{3}$ then any descending sequence has $k(f) \leq 1$ and the optimal probability of nonextinction is

$$
P_{0}(f)= \begin{cases}p f, & f \leq \eta, \\ 1-(\sqrt{|c|(1-p)}+p \sqrt{1+a-f})^{2}, & \eta<f<1, \\ 1, & f=1 .\end{cases}
$$

In addition, $1-P_{0}(1-)=[\sqrt{|c|(1-p)}+p \sqrt{a}]^{2}>0$ and $P_{0}(f)$ has continuous derivative for $0 \leq f<1$.

Proof. We want to calculate $R(f)=1-P(f)$ :

$$
R(f)=\inf _{f_{1}}\left(1-p \frac{f-(1+a) f_{1}}{1-f_{1}}\right)\left(1-p f_{1}\right)=\inf _{f_{1}} U\left(f_{1}\right),
$$

where $0 \leq f_{1} \leq(1+a)^{-1} f$.

The function $U$ to be minimized in $f_{1}$ is convex on the interval of interest, $[0,1)$. We recall that strict bold play, when we bet $s=f$ for all $f$, results in the minimum being realized at $f_{1}=0$ for all $f$, which we shall see is not the case. Rewriting, we obtain

$$
U(x)=|c|(1-p x)+p(1-p)(1+a-f)(1-x)^{-1}+p^{2}(1+a-f),
$$

whence

$$
U^{\prime}(x)=p(1-p)(1+a-f)(1-x)^{-2}-p|c|
$$

in particular,

$$
U^{\prime}(0)=p \frac{a+|c|}{1+a}\left(\frac{a+a^{2}}{|c|+a}-f\right), \quad U^{\prime}\left(\frac{f}{1+a}\right)>0 .
$$

Given that $f>\eta=\left(a+a^{2}\right) /(a+|c|)$, we have $U^{\prime}(0)<0$, showing that the minimizer $x=f_{1}^{-}$is in $(0, f /(1+a))$. The exact value is

$$
f_{1}^{-}(f)=1-\sqrt{\left(1-\frac{f}{1+a}\right)\left(1+\frac{a}{|c|}\right)}
$$

providing the exact strategy

$$
s(f)= \begin{cases}f-(1+a) f_{1}^{-}(f), & f>\left(a+a^{2}\right) /(a+|c|), \\ f, & f \leq\left(a+a^{2}\right) /(a+|c|) .\end{cases}
$$

It is easy to verify the value of the jump discontinuity at $f=1$ and the equality of the one-sided limits at $\eta$. 


\section{Calculations for $k(f)=2$}

To gain some insight into the computational difficulty of the case in which the descending sequence used in (11) and (12) involves more than two terms, we investigate the simplest case, in which $k(f)=2$ (the sequence length is at least three).

We have to evaluate

$$
\inf _{f_{1}, f_{2}}\left(1-p \frac{f-(1+a) f_{1}}{1-f_{1}}\right)\left(1-p \frac{f_{1}-(1+a) f_{2}}{1-f_{2}}\right)\left(1-p f_{2}\right)
$$

with $f_{1} \leq(1+a)^{-1} f$ and $f_{2} \leq(1+a)^{-1} f_{1}$. An alternative expression, based on (14), is

$$
\inf _{\left(\gamma_{0}, \gamma_{1}\right) \in(0,1] \times(0,1]} \frac{\left(1+a-\gamma_{0}\right)\left(1+a-\gamma_{1}\right)+\gamma_{0}-p f}{\left(1+a-\gamma_{0}\right)\left(1+a-\gamma_{0}\right)}\left(1-p \gamma_{0}\right)\left(1-p \gamma_{1}\right),
$$

but we proceed using (19). We are interested in nontrivial (interior) critical points $f_{1}=f_{1}^{\prime}$ and $f_{2}=f_{2}^{\prime}$ of the function (19), since the boundary cases correspond to $k \leq 1$. We make the observation that if the function

$$
x \rightarrow\left(1-p \frac{g-(1+a) x}{1-x}\right)(\rho-x), \quad x \in[0,1), \quad \rho \geq 1,
$$

has a nontrivial critical point $x^{\prime} \in(0,1)$, then this point takes the value

$$
x^{\prime}=1-\sqrt{(\rho-1)\left(\frac{1}{|c|}-1\right)\left(1-\frac{g}{1+a}\right)} .
$$

By fixing $f_{2}$ in (19) and applying (20) and (21) with $g=f, \rho=\left(1+c f_{2}\right) p^{-1} \geq 1$, and $x=f_{1}$, and then fixing $f_{1}$ in (19) and applying (20) and (21) with $g=f_{1}, \rho=p^{-1} \geq 1$ and $x=f_{2}$, we obtain the system of equations

$$
\begin{aligned}
1-f_{1}^{\prime} & =\sqrt{\left(1+\frac{a}{|c|}-(1+a) f_{2}^{\prime}\right)\left(1-\frac{f}{1+a}\right)}, \\
1-f_{2}^{\prime} & =\sqrt{\left(1+\frac{a}{|c|}\right)\left(1-\frac{f_{1}^{\prime}}{1+a}\right)}
\end{aligned}
$$

equivalent to finding a real zero of a polynomial of degree four.

\section{An upper bound}

An upper bound for the probability of success is

$$
P_{1}(f)=1-(1-f)^{(1+c) /(1+a)},
$$

for any initial fortune $f, 0 \leq f \leq 1$.

Remark 3. We note that $p=(1+c) /(1+a)$ is such that $0<p<1$, and any function $1-(1-f)^{p^{\prime}}$ with $p^{\prime} \geq p$ provides an upper bound.

Proposition 8. Let $\left\{X_{n}^{S}\right\}_{n \geq 0}$ be the chain describing the evolution of the gambler's fortune, satisfying (2), with initial value $X_{0}=f$. Then, for any strategy $S \in \mathcal{S}$, the process $\left\{P_{1}\left(X_{n}\right)\right\}_{n \geq 0}$ is a supermartingale with respect to $\left\{\mathcal{F}_{n}\right\}_{n \geq 0}$. 
Proof. Recall that the winning probability, $w(c, r)$, is equal to $(1+c) /(1+r)$. The reasoning is identical to that leading to the upper bound from Theorem 1, obtained via Proposition 6 . We have to prove the analogue of (16) for the utility function (22), that is,

$$
P_{1}\left(\frac{f+r s}{1+a}\right) w(c, r)+P_{1}\left(\frac{f-s}{1+a}\right)(1-w(c, r)) \leq P_{1}(f)
$$

for $0 \leq f \leq 1,0 \leq s \leq f, f+r s \leq 1+a$, and $r \geq 0$. This inequality is equivalent to

$$
\left(1-\frac{f+r s}{1+a}\right)^{p} \frac{1+c}{1+r}+\left(1-\frac{f-s}{1+a}\right)^{p}\left(1-\frac{1+c}{1+r}\right) \geq(1-f)^{p} .
$$

We think of (23) as a function of $s$ and $f$ with $a$ and $r$ fixed.

For fixed $f$, the left-hand side of (23) is a concave function of $s$, which shows that it is sufficient to check its values at the endpoints. We write

$$
\begin{aligned}
\psi(s) & =\left(1-\frac{f}{1+a}-\frac{r}{1+a} s\right)^{p} w(c, r)+\left(1-\frac{f}{1+a}+\frac{1}{1+a} s\right)^{p}(1-w(c, r)) \\
& =\left(c_{1}-c_{2} r s\right)^{p} w+\left(c_{1}+c_{2} s\right)^{p}(1-w)
\end{aligned}
$$

with $c_{1}=1-f /(1+a)$ and $c_{2}=1 /(1+a)$. The derivative with respect to $s$,

$$
p\left(c_{1}-r c_{2} s\right)^{p-1}\left(-c_{2} r\right) w+p\left(c_{1}+c_{2} s\right)^{p-1} c_{2}(1-w),
$$

is decreasing, proving that $\psi(s)$ is concave.

It remains to verify (23) at the endpoints of the interval where $s$ is compatible in the sense of (4). The restrictions on $s$ and $f$ split the problem into two cases: case 1, in which $a<r$, and case 2 , in which $a \geq r$. Case 1 is further split into case 1 (i), in which $f \geq(1+a) /(1+r)$, implying that $0 \leq s \leq(1+a-f) / r$, and case 1(ii), in which $f \leq(1+a) /(1+r)$, implying that $0 \leq s \leq f$.

Cases 1(i), 1(ii), and 2 with $s=0$. The inequality is trivial in these cases.

Case 1(i) with $s \neq 0$. We have to check (23) for $s=(1+a-f) / r$, i.e.

$$
\left(1-\frac{f-(1+a-f) r^{-1}}{1+a}\right)^{p}(1-w) \geq(1-f)^{p} .
$$

The inequality is trivially true for $f=1$. Divide through by the right-hand side; we then have to show that

$$
\left(1+\frac{1}{r}\right)^{p}\left(\frac{1-f /(1+a)}{1-f}\right)^{p}(1-w) \geq 1 .
$$

The function in $f$ is increasing, so it suffices to prove the inequality for $f=(1+a) /(1+r)$, i.e. to prove that

$$
1-\frac{1+c}{1+r} \geq\left(1-\frac{1+a}{1+r}\right)^{p}
$$

for $r \geq 0$. This is a consequence of the fact that $(1-p x) \geq(1-x)^{p}$ for all $x \geq 0$, with $x=1 /(1+r)$ and $p=(1+c) /(1+a)$.

Case 1(ii) with $s \neq 0$. We have to check (23) for $s=f$, i.e.

$$
\left(1-\frac{1+r}{1+a} f\right)^{p} w+(1-w)-(1-f)^{p} \geq 0
$$


with $f \leq(1+a) /(1+r)$. We can see that the derivative of the function of $f$ on the left-hand side changes sign only once in the interval, from a positive value to a negative value. This shows that the minimum is to be found at $f=0$ or $f=(1+a) /(1+r)$. It is sufficient to verify that

$$
1-\frac{1+c}{1+a} \geq\left(1-\frac{1+a}{1+r}\right)^{p},
$$

which holds as shown above.

Case 2 with $s \neq 0$. In this case we have to check (23) for $s=f$, as in case 1(ii), which means verifying (24) for $0 \leq f \leq 1$. Again, with the same reasoning, we find that the minimum is reached at either $f=0$ or $f=1$. For $f=0$ the left-hand side of (23) equals 0 , and for $f=1$ we obtain

$$
\left(1-\frac{1+r}{1+a}\right)^{p} w+(1-w)>0 .
$$

\section{Acknowledgements}

The authors would like to express their gratitude to the anonymous referees for the careful reading of the paper and their valuable comments.

\section{References}

[1] Chen, R. (1977). Subfair primitive casino with a discount factor. Z. Wahrscheinlichkeitsth. 39, 167-174.

[2] Chen, R. (1978). Subfair 'red-and-black' in the presence of inflation. Z. Wahrscheinlichkeitsth. 42, $293-301$.

[3] Chen, R. W., Shepp, L. A. And Zame, A. (2004). A bold strategy is not always optimal in the presence of inflation. J. Appl. Prob. 41, 587-592.

[4] Chen, R. W., Shepp, L. A., Yao, Y.-C. and Zhang, C.-H. (2005). On optimality of bold play for primitive casinos in the presence of inflation. J. Appl. Prob. 42, 121-137.

[5] Coolidge, J. L. (1909). The gambler's ruin. Ann. Math. 10, 181-192.

[6] Dubins, L. E. And Savage, L. J. (1965). How to Gamble if You Must. Inequalities for Stochastic Processes. McGraw-Hill, New York.

[7] Heath, D. C., Pruitt W. E. And Sudderth, W. D. (1972). Subfair red-and-black with a limit. Proc. Amer. Math. Soc. 35, 555-560.

[8] Klugman, S. (1977). Discounted and rapid subfair red-and-black. Ann. Statist. 5, 734-745.

[9] Lou, J. (2007). A paradox: having more choice of games in a casino provides little advantage. Doctoral Thesis, Rutgers University.

[10] Maitra, A. P. And Sudderth, W. D. (1996). Discrete Gambling and Stochastic Games (Appl. Math. (New York) 32). Springer, New York.

[11] Rockafellar, R. T. (1997). Convex Analysis. Princeton University Press.

[12] Shepp, L. A. (2006). Bold play and the optimal policy for Vardi's casino. In Random Walks, Sequential Analysis and Related Topics, eds C. A. Hsiung, Z. Ying and C.-H. Zhang, World Scientific, Singapore, pp. 285-291.

[13] VARDI, Y. (2001). Private communication. 